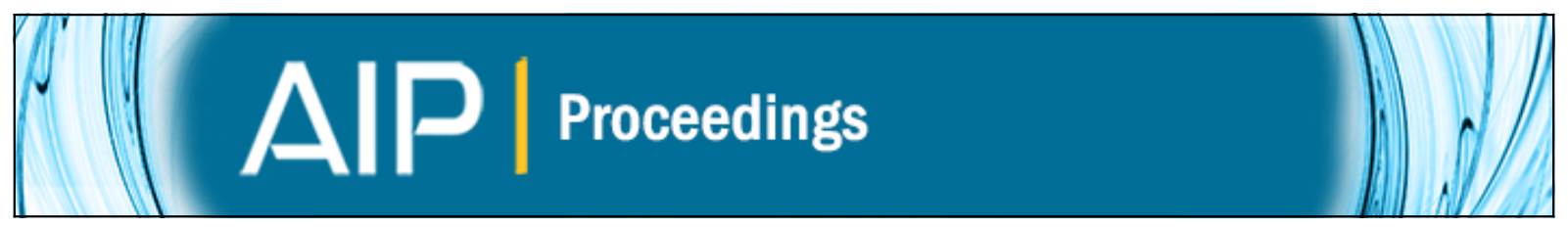

Wavelet Analysis of Spatiotemporal Network Oscillations Evoked in the Incilaria Brain

A. Schütt, O. A. Rosso, Y. Makino, T. Fujie, M. Yano, M. Werner, A. Figliola, and U. G. Hofmann

Citation: AIP Conference Proceedings 913, 209 (2007); doi: 10.1063/1.2746749

View online: http://dx.doi.org/10.1063/1.2746749

View Table of Contents: http://scitation.aip.org/content/aip/proceeding/aipcp/913?ver=pdfcov

Published by the AIP Publishing

Articles you may be interested in

Seizure-induced alterations in fast-spiking basket cell GABA currents modulate frequency and coherence of gamma oscillation in network simulations

Chaos 23, 046109 (2013); 10.1063/1.4830138

Theoretical and experimental analysis of the forced Lacl-AraC oscillator with a minimal gene regulatory model Chaos 23, 025109 (2013); 10.1063/1.4809786

Sensory coding in oscillatory electroreceptors of paddlefish

Chaos 21, 047505 (2011); 10.1063/1.3669494

Spatiotemporal dynamics of networks of excitable nodes

Chaos 16, 015110 (2006); 10.1063/1.2177569

Noise-induced Synchronized Oscillations in an Inhibitory Neural Network

AIP Conf. Proc. 665, 191 (2003); 10.1063/1.1584891 


\title{
Wavelet Analysis of Spatiotemporal Network Oscillations Evoked in the Incilaria Brain.
}

\author{
A. Schütt*, O. A. Rosso ${ }^{\dagger}$, Y. Makino**, T. Fujie**, M. Yano**, M. Werner*, \\ A. Figliola ${ }^{\ddagger}$ and U. G. Hofmann* \\ *Institute for Signal Processing, University of Lübeck. \\ Ratzeburger Allee 160, 23538 Lübeck, Germany. \\ $\dagger$ Instituto de Cálculo, Facultad de Ciencias Exactas y Naturales, Universidad de Buenos Aires. \\ Pabellon II, 1428 Ciudad Universitaria, Buenos Aires, Argentina. \\ ${ }^{* *}$ Research Institute of Electrical Communication, Tohoku University. \\ 2-1-1 Katahira, Aoba-ku, Sendai-shi, 980-8577 Japan. \\ $\$$ Instituto del Desarrollo Humano, Universidad Nacional de General Sarmiento. \\ Juan Maria Gutierrez 1150, Los Polvorines, Pcia. de Buenos Aires, Argentina.
}

\begin{abstract}
In the slugs and snails odor input signal, partly processed by the tentacle ganglion, propagates through the tentacle nerve (TN) to the cerebral ganglion, initially activating the meso-meta-region and finally the procerebral region (PC). The PC, equivalent to mammalian olfactory bulb, exerts slow spontaneous neuroelectrical oscillation, which changes its frequency and amplitude pattern responding to stimulus input. This has been related to a mechanism of signal processing for odor encoding. Three neuronal substructures, the cell mass (CM), the terminal mass (TM) and internal mass (IM) form the PC. Records from IM and CM have extensively been studied, but those from TM have scarcely been investigated. In the present study we aimed to clarify network dynamics among these cell ensembles with particular interest in the property of TM. Methods: We isolated the cerebral ganglia from the slug Incilaria together with TNs. We applied to TN electrical stimulation of weak to strong intensities $(0.1-1.0 \mu \mathrm{A})$ and recorded activities at the three loci of PC by glass suction electrodes at a sampling rate of $200 \mathrm{~Hz}$. The data were stored on hard drive and later off-line analysed by wavelet tools. Results: Wavelet analysis revealed that the major power of the spontaneous oscillations laid below $1.6 \mathrm{~Hz}$. Namely, in the Incilaria PC, mainly the frequency components $<1.6 \mathrm{~Hz}$ take part in the dynamical signal processing. The frequency components, that are timedependently, interacting with each other, contribute together to altering total entropy of a cell mass at a given time. Notably, the $0.1-0.2 \mathrm{~Hz}$ component contributing most strongly to total energy attributes most to dropping entropy ("ordering of neuronal state"). Response to the weakest stimulus is most sensitively elicited as "desynchronization" in TM-IM, but that to the stronger stimuli, as "synchronization or frequency ordering" in TM-CM, and finally "synchronization" in TM-IM-CM (the whole PC). The fact that the entropy of TM in general remains lower than IM and CM regardless with stimulation suggests that the neurons of TM are in more ordered state than the other masses playing some governing function in the procerebral network.
\end{abstract}

Keywords: Incilaria procerebrum, electrical stimulation, wavelet transform, entropy. PACS: 7.19.La, 89.75.Fb

\section{INTRODUCTION}

The brains (the circumesophageal ganglion complex) of the molluses (snails, slugs) have been favoured by neuroscientists as easily accessible animal models. In paticular, the olfactory system (neuroepithelium of tentacle gangliontentacle nerve-procerebral lobe of cerebral ganglion) is known to be anatomically very similar to that of the mammals [1] and has for this reason been favoured for study on olfaction.

The procerebral lobe intrinsically exerts slow local field potential oscillation at $0.6-1.0 \mathrm{~Hz}$ and this oscillation changes its frequency and amplitude pattern when stimulation, such as odor, is applied to the nose (neuroepithelium). This phenomenon is presumed to be related to signal processing for odor encoding by the brain and has been extensively studied in conjunction with the general question on the mechanism of odor information processing $[2,3,4,5]$.

How does the signal processing proceed within such brain network (the cerebral ganglion consisting of the meso, meta- and pro-cerebrum)? It has been presumed that in the slugs and snails input signal, first partly processed by the tentacle ganglion, propagates through the tentacle nerve (TN) to the meso-meta-regions of the cerebral ganglion where initial activation takes place. The activation then finally spreads to the procerebral region (PC) for further

CP913, Nonequilibrium Statistical Mechanics and Nonlinear Physics, XV Conference

edited by O. Descalzi, O. A. Rosso, and H. A. Larrondo

C 2007 American Institute of Physics 978-0-7354-0421-2/07/\$23.00 


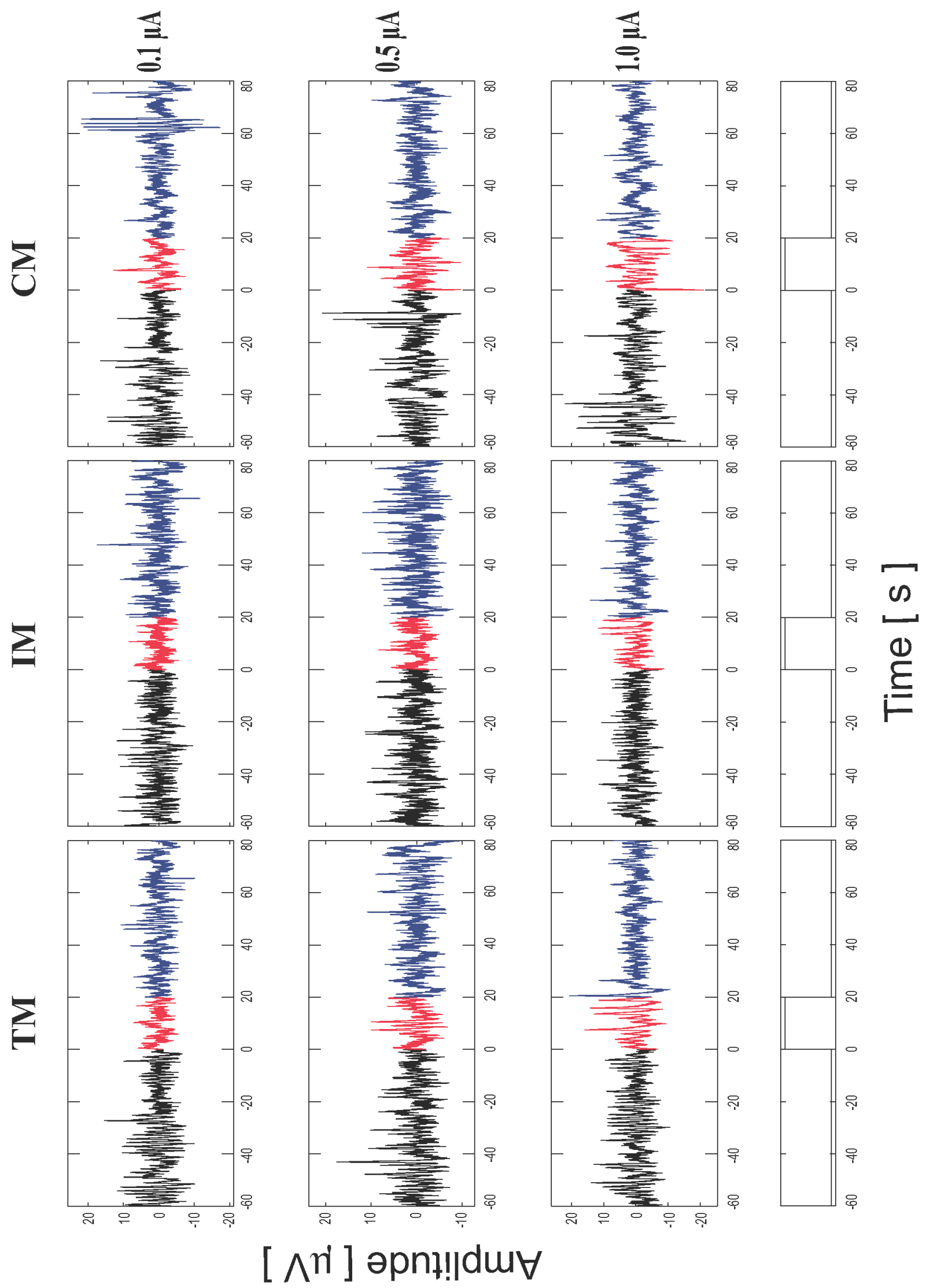

FIGURE 1. Time signals of a typical experiment. 
processing. Our recent study with a voltage-sensitive dye method [4] has for the first time evidenced that on stimulus input (electrical stimulation of the tentacle nerve) initial response takes place in the meso- and metacerebral regions and then propagates to the procerebral region eventually activating the whole cerebral ganglion. The electrophysiological measurements applying odor stimuli have also evidenced this. However, the spatiotemporal dynamics within the procerebrum (PC) itself is yet to be clarified.

The PC is constructed from three neuronal substructures, the cell mass (CM), the terminal mass (TM) and internal mass (IM). Records from CM and IM have widely been studied, but those from TM have not systematically been investigated. We presume that TM may have some important functional role.

In our previous studies $[6,7]$ we have demonstrated that it is possible to describe discrete, stimulus evoked change of neuronal state of such cell ensembles by Wavelet analysis. In the present study we aimed to find out by applying these methods, with particular interest on the property and role of TM, how signal processing takes place among the cell ensembles of the procerebral network.

\section{METHODS}

Slugs (Incilaria fruhstorferi) weighing $2.5-9.6 \mathrm{~g}$ were used. The animals fasted overnight before preparation. We isolated the whole cerebral ganglion under anaesthesia by cooling with crashed ice for $40 \mathrm{~min}$. The preparation included the superior tentacle ganglia and the tentacle nerves as well as the inferior tentacle ganglia and the lip nerves. It was then fixed in a recording chamber filled with slug saline (in mM: $39.4 \mathrm{NaCl} ; 1.8 \mathrm{KCl} ; 4.0 \mathrm{CaCl} 2$; 3.7 MgCl2; 5.0 glucose; 2.36 HEPES; 2.64 HEPES Na). For stimulation we applied weak to strong electrical currents $(0.1-1.0 \mu \mathrm{A} ; 20 \mathrm{~s})$ to the tentacle nerve and recorded the local field potential activities from the three loci $(\mathrm{CM}, \mathrm{TM}$, IM) simultaneously using glass suction electrodes $(\mathrm{Ag}-\mathrm{AgCl})$. For each trial a $60 \mathrm{~s}$ prestimulus; $20 \mathrm{~s}$ peristimulus and $60 \mathrm{~s}$ poststimulus epochs were recorded at a sampling rate of $200 \mathrm{~Hz}$. Digitised data were stored in hard drive and later off-line analysed by the wavelet tools. "Relative Wavelet Energy (RWE)" describes contribution of each frequency component energy to the total energy at a given time and "Normalized Total Wavelet Entropy (NTWS)" determines "degree of ordering or synchronization" of a cell ensemble at a given time. The mathematical background can be referred to our earlier reports $[6,7,8]$.

\section{RESULTS}

In Fig. 1 we present time signals of a typical experiment. Electrical stimulation of the tentacle nerve altered the patterns of the ongoing rhythmical oscillations of all masses. At the weakest stimulus $0.1 \mu \mathrm{A}$ suppression of the oscillatory waves were visible in TM and IM, but at higher stimulus intensities either burst of oscillatory waves, which were clearly slower than the prestimulus waves, or typical evoked potential wave forms were observed.

Wavelet analysis was performed on each of all trials and on the grand average for each of CM, IM and TM and for each of stimulus intensities. In the present study we chose six bands $\left(B_{10}=0.1-0.2 \mathrm{~Hz} ; B_{9}=0.2-0.4 \mathrm{~Hz}\right.$; $\left.B_{8}=0.4-0.8 \mathrm{~Hz} ; B_{7}=0.8-1.6 \mathrm{~Hz} ; B_{6}=1.6-3.2 \mathrm{~Hz} ; B_{5}=3.2-6.4 \mathrm{~Hz}\right)$ for the analysis. In Fig. 2 we show the grand average ( $N=33$ ) of Relative Wavelet Energy (RWE). These results reveal the following: $a$ ) The major power of the spontaneous oscillations lay below $1.6 \mathrm{~Hz}$. Mainly four frequency components $B_{10}-B 7(0.1-0.8 \mathrm{~Hz})$ contribute to the formation of the procerebral oscillation. b) Component energy distribution dynamically changes with time and event. c) Contribution of the $B_{1}(0.1-0.2 \mathrm{~Hz})$ component energy is particularly high (up to $\left.50 \%\right)$ and response to stimulation took place most conspicuously in this component energy. d) Between the $B_{10}(0.1-0.2 \mathrm{~Hz})$ component and the $B_{8}-B_{7}(0.4-1.6 \mathrm{~Hz})$ component a negative correlation seems to exist. Note particularly that in TM and IM stimulation at $0.1 \mu \mathrm{A}$ caused a decrease of the $B_{10}(0.1-0.2 \mathrm{~Hz})$ component energy. The weak stimulus-evoked suppression in TM and IM (Fig. 1) may be explained by this.

In Fig. 3 we display the grand average $(N=33)$ of Normalized Total Wavelet Entropy (NTWS). The curves show time-dependent, dynamical changes of neuronal states of the cell ensembles, and seem to differentiate, stimulusdependently, response behaviour of each neuronal mass. For instance, TM and IM responded to the weakest stimulus, $0.1 \mu \mathrm{A}$, increasing the entropy ("neuronal disorganization"). As the stimulus intensity was increased $(0.5 \mu A), \mathrm{CM}$ elicited a strong response with a sudden, large drop of entropy ("neuronal synchronization"), and this appeared to be coupled with the similar, but slightly less, entropy drop of TM. As for IM, the delayed slight decrease of entropy is largely due to the delayed increase of the $B_{10}$ component energy. By the strongest stimulus $(1.0 \mu \mathrm{A})$ entropy dropped in all masses, which suggested "a synchronized activity" throughout the whole PC. Survey of RWE curves indicates 

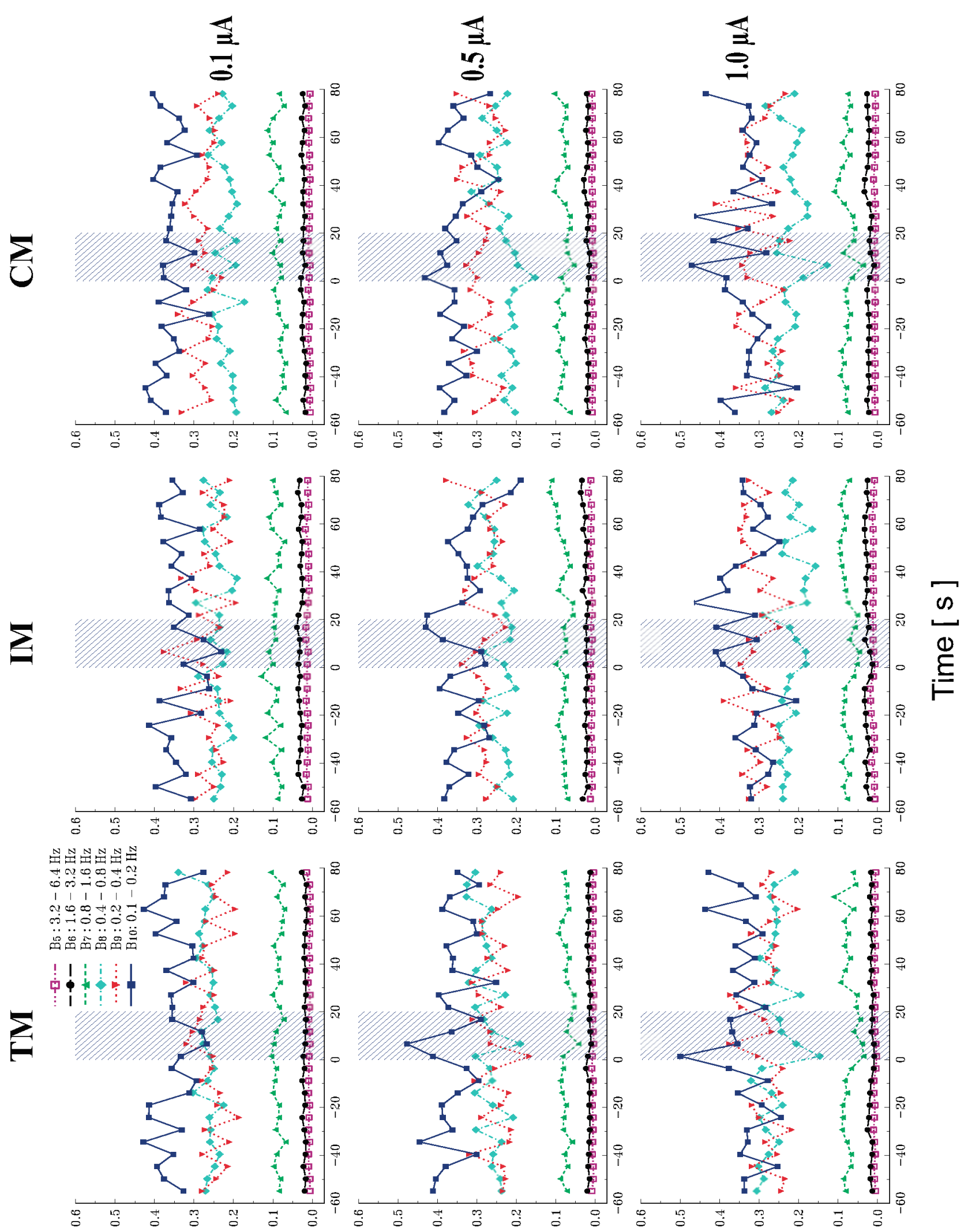

\section{Кбләи ґәрәлем әм!ฺе|әу}

FIGURE 2. Grand average $(N=33)$ of Relative Wavelet Energy (RWE) as tine fuction. Shadowed period: stimulation (20 $s$ ). 


\section{Normalized Total Wavelet Entropy}

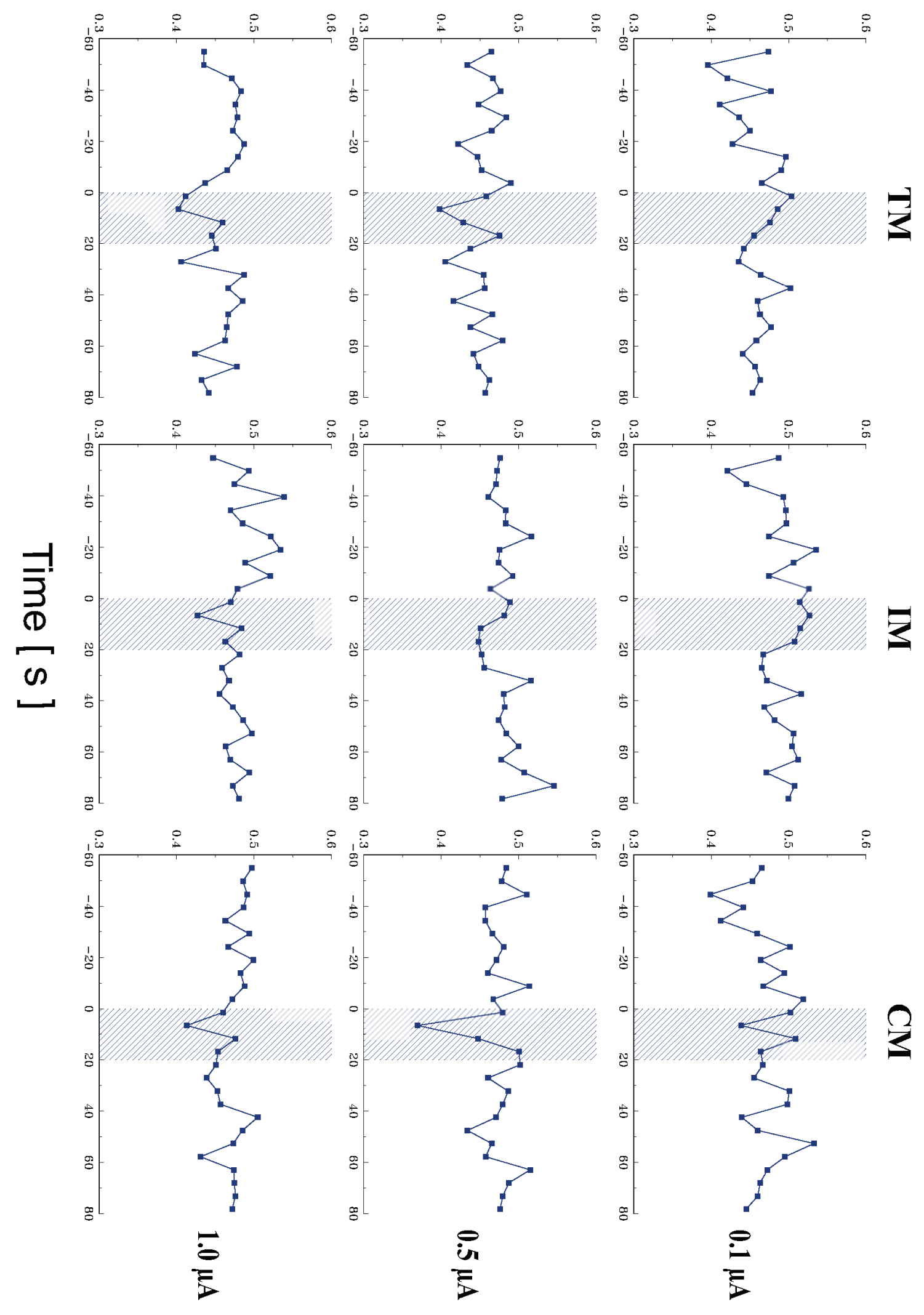


that the sharp entropy drop is mainly attributed to the strong $B_{10}(0.1-0.2 \mathrm{~Hz})$ component energy increase as well as the $0.2-0.4 \mathrm{~Hz}$ and $0.4-0.8 \mathrm{~Hz}$ energy decreases (Fig. 2).

These results based on entropy may suggest that the stimulus at weakest intensity causes desynchronization in TMIM, but as the intensity increases, synchronization in TM-CM and in IM with a delay, and finally synchronization in TM-IM-CM (the whole PC). Another important finding is that entropy of TM fluctuated at a slightly lower level in all grand averages compared with those of IM and CM. This would indicate that the neuronal ensemble of TM remained in more ordered state in general than in those of IM and CM. Most importantly, the frequency components that are interacting with each other (Fig. 2) contribute together to altering total entropy of a cell mass.

\section{DISCUSSION}

In the present study we aimed to characterize the oscillatory network dynamics among three cell ensembles of the Incilaria procerebrum by electrophysiological recording and application of wavelet analyses. The fact that the entropy of TM in general remains lower than those of IM and CM regardless with stimulation suggests that the neurons of TM are in more ordered state than those of the other masses playing some governing function in the procerebral network.

The responses observed in the present study were caused by artificial stimuli, but were very similar to what we found earlier in the procerebrum of the snail Helix pomatia using odor stimuli: $i$ ) the neuronal disorganization evoked in TM and IM by a weak current resembles that evoked by an attractive odor. ii) strong entropy drop attributed to the increase of the $0.1-0.2 \mathrm{~Hz}$ component energy also occurs by stimulation with aversive odor [5].

\section{ACKNOWLEDGMENTS}

This work was supported by the Research Institute of Electrical Communication, Tohoku University, Sendai, Japan (grant for visiting professorship, No. 2004-561608-2). This work was also supported by Consejo Nacional de Investigaciones Científicas y Técnicas (CONICET) (PIP 5687/05), Agencia Nacional de Promoción Cientifica y Tecnológica (PICT 20648/04). The authors thank Kaarina M. Meyn for her skilful assistance in preparation of the graphical layouts.

\section{REFERENCES}

1. R. Chase, B. Tolloczko, Microsci. Res, Technique 24, 214-230 (1993).

2. A. Gelperin, D. W. Tank, Nature 435, 437-440 (1990).

3. T. Kimura, S. Toda, T. Sekiguchi, Y. Kirino, Learning \& Memory 4, 365-375 (1998).

4. H. Makinae, Y. Makino, M. Yano, Zool. Sci. 20, 1580-1588(2003)

5. A. Schütt, E. Başar, T. H. Bullock, Comp. Biochem. Physiol. Part A 123, 95-110 (1999).

6. A. Schütt, O. A. Rosso, A. Figliola, J. Neurosci. Methods 119, 89-104 (2002).

7. A. Schütt, I. Ito, O. A. Rosso, A. Figliola, J. Neurosci. Methods 129, 135-150 (2003).

8. O. A. Rosso, S. Blanco, J. Yordanova, V. Kolev, A. Figliola, M. Schr̈mann, E. Başar, J. Neurosci. Methods 105, 65-75 (2001). 